\title{
Electron tunneling in the presence of adsorbed molecules
}

\author{
R. C. Hoft, M. J. Ford*, and M. B. Cortie \\ Institute for Nanoscale Technology, University of Technology Sydney, PO Box 123, Broadway NSW 2007, Australia
}

(Received )

We perform $a b$-initio density functional theory calculations of the tunneling current through an electrodemolecule-electrode system with four different small organic molecules, benzenedithiol (BDT), benzenedimethanethiol (XYL), diethynylbenzene (DEB) and dodecanethiol (C12), sandwiched between two gold (111) electrodes. For the XYL molecule we test the effect of alternate bonding types and sites. Although this reduces the current considerably, it does not account for the orders of magnitude differences between experimental and theoretical results in the literature. We also model a typical STM experimental setup with a gold nanoparticle absorbed on a self-assembled monolayer (SAM) of the molecule with a gap between the nanoparticle and probing tip and show that such a gap could account for these differences. Finally we describe the effect the gap has on the ability of STS measurements to distinguish between the $i(V)$ characteristics and thicknesses of self assembled monolayers of different molecules.

Keywords: Molecular electronics, single molecule conductance, electron transport, ab-initio transport calculations.

PACS numbers: 73.63.-b, 73.40.Gk, 85.35.-p

\section{Introduction}

Studies of electron tunneling between two electrodes with adsorbed bridging molecules are attracting significant attention due to possible application of this phenomenon in the fabrication of an electronic device based on active components consisting of molecules [1]. It is now firmly established that the response of such a system to an applied bias between the electrodes does not only depend on the geometric and electronic structure of the molecules itself, but also to a large extent on the nature of the coupling between the molecule and the electrodes [2]. It is believed that this point could be the main reason for discrepancies that exist between experimental and theoretical data. 
The formation of self-assembled monolayers of thiolate molecules on gold surfaces is well studied [3] and it is believed that the gold-sulfur interaction is comparatively strong. For this reason the gold-thiolate-gold system is often used for conductance measurements and theoretical calculations. Perhaps the first experimental measurement of the conductance of such a system was performed by Reed et. al. [4] where the setup is achieved by forming a mechanically controllable break junction. The authors measured the conductance near 0 volt of a gold-benzenedithiol-gold system as $0.045 \mu \mathrm{S}$. Cui et. al. [5] measured an i(V) curve for a goldoctanedithiol-gold system by driving an AFM tip into gold nanoparticles which were used to activate octanedithiol molecules inserted into an octanethiol monolayer on a $\mathrm{Au}(111)$ surface. In the Ohmic region between $-0.1 \mathrm{~V}$ and $0.1 \mathrm{~V}$ they measured a conductance of $0.0011 \mu \mathrm{S}$. Tao et. al. [6, 7] used an STM technique to measure the conductances near 0 volt of benzenedithiol, benzenedimethanethiol, 4,4' bipyridene, hexanedithiol, octanedithiol and decanedithiol between gold electrodes as $0.85 \mu \mathrm{S}, 0.046 \mu \mathrm{S}, 0.77 \mu \mathrm{S}, 0.095$ $\mu \mathrm{S}, 0.019 \mu \mathrm{S}$ and $0.0016 \mu \mathrm{S}$ respectively.

Previous ab-initio DFT studies of benzenedithiol between $\mathrm{Au}(111)$ electrodes have resulted in conductances up to $40 \mu \mathrm{S}$ [8]. This is typical of theoretical calculations which tend to overestimate current through molecules by one or two orders of magnitude [2]. We believe discrepancies between experimental and theoretical results and indeed also between different experimental results can largely be attributed to the contact geometry at the molecule-electrode interface. This geometry is ill defined experimentally. The most common theoretical setup is to assume a perfect contact geometry with the sulfur atom of the thiolate molecule placed in a hollow site on the $\mathrm{Au}(111)$ surface on each electrode. Recently calculations have been done with consideration for alternate sulfur binding sites [9-11] and intermediate gold atoms between the sulfur atom and $\mathrm{Au}(111)$ surface [12].

Other issues relating to the disparity between theoretical and experimental results have been investigated, including the representation of infinite electrodes with finite-dimensional methods [11], the possible open-shell nature of the system for which a restricted open-shell treatment is more appropriate [13], and self-interactions errors occurring in the weak-coupling limit [14]. More accurate descriptions of throughmolecule transport can be obtained from advanced theories such as the configuration interaction formalism 
[15] to account for electron correlation, or time-dependent DFT methods [16]. These are beyond the scope of the present work.

In this paper, we perform ab-initio calculations of the $i(V)$ curves of benzenedithiol (BDT), benzenedimethanethiol (XYL), diethynylbenzene (DEB) and dodecanethiol (C12) between Au(111) electrodes in the usual idealised bonding geometry. For the XYL molecules we check the effect of different binding sites and a different bonding type on one electrode. In a further effort to reconcile theoretical and experimental results, we introduce a tunnel gap between molecule and electrode on one end in an attempt to simulate a scanning tunneling spectroscopy (STS) measurement of $i(V)$ characteristics. This can be troublesome in $a b$ initio calculations and therefore we introduce a gold nanoparticle absorbed on the monolayer and rather model the effect of a tunnel gap between this nanoparticle and the electrode, which we calculate empirically. This setup is similar to the experiments of Cui et. al. [5].

\section{Calculation}

The SIESTA $[17,18]$ density functional code was used to perform geometry optimisations to obtain the ideal bonding geometry of the molecules on a $\mathrm{Au}(111)$ surface. The code employs a linear combination of numerical atomic orbitals as basis functions. We use double zeta plus polarization basis sets for the valence electrons. These orbitals have finite extent in space and are cut off at a radius $r_{c}$ corresponding to an excitation energy due to the confinement of $5 \mathrm{mRy}$. The core electrons are modeled using norm-conserving pseudopotentials constructed according to the scheme of Troullier and Martins [19]]. Relativistic corrections to the pseudopotentials are made for the gold atoms. The exchange-correlation energy is calculated with the generalized gradient approximation (GGA) using the parameterization of Perdew, Burke and Ernzerhof [20]]. The $\mathrm{Au}(111)$ surface is modeled using a 4 layer slab with $3 \times 3$ gold atoms per layer. This is repeated periodically in all three directions with a unit cell length in the direction normal to the slab of $40 \AA$, which ensures that the slabs are non-interacting. These computational conditions, together with the pseudopotentials employed have been tested rigorously by us and result in well-converged energies [21, 22] The transport calculations were undertaken with the TRANSIESTA-C [23]] package, originally based on SIESTA. This 
code finds the electronic structure in the same way as SIESTA and the non-equilibrium Green's functions technique (NEGF) is used to calculate the current when a bias is applied between the two opposite electrodes. The code does not implement the generalized gradient approximation ${ }^{1}$ and therefore the local density approximation (LDA) to exchange and correlation is used with the parameterization of Perdew and Zunger [24]]. For the gold atoms we used a single zeta plus polarization basis and the orbital confinement is defined with an excitation energy of $10 \mathrm{mRy}$ for all atoms. Comparison with a calculation using double zeta plus polarization orbitals on gold atoms and a $5 \mathrm{mRy}$ excitation energy showed only a small change in the transmission spectrum at a 0 volt bias. The other parameters are the same as described above for SIESTA. TRANSIESTA-C works by finding the density matrix in the bulk electrode parts with a separate calculation. Here we include two layers of $\mathrm{Au}(111)$ in the central ("extended molecule”) region so that the approximation of bulk electronic structure is correct from the third layer onwards (see Fig. 1). The bonding geometry on each side is constructed according to the lowest energy configuration found with SIESTA.

For the STS configuration shown in Fig 2 we assume that the gold nanoparticle is sufficiently large that coulomb blockade effects can be ignored and that the tunneling current in the nanoparticle-moleculeelectrode region can be calculated exactly as above using the NEGF method. The current through the gap between the tip and nanoparticle can be calculated using the Wentzel-Kramers-Brillioun (WKB) approximation. From tunneling through rectangular barriers, where an analytical solution can be found, it is known that the WKB gives a good approximation to the exact solution provided the electron energy is much smaller than the barrier height. This is the case in the present work, and we can be confident that the WKB will give reasonable results. The transmission function is now given by

$$
T(E, V, d)=e^{\frac{2 \sqrt{2}}{\hbar} \int_{0}^{d} \sqrt{m(U(x)-E)} d x}
$$

\footnotetext{
${ }^{1}$ A later version of the code made available recently does implement GGA and has been renamed ATK 2.0. The code is available from Atomistix A/S (see www.atomistix.com).
} 
where $m$ is the electron mass, $d$ is the width of the gap and $U(x)$ is the potential function across the gap. The current is then found from the Landauer formalism as

$$
i(V, d)=\frac{2 e}{h} \int_{-\infty}^{\infty} T(E, V, d) \rho_{t}(E-e V) \rho_{p}(E)[f(E)-f(E-e V)]
$$

where $\rho_{t}$ and $\rho_{\mathrm{p}}$ are the local densities of states of the tip and particle, $V=V_{\text {particle }}-V_{\text {tip }}$ is the bias applied between tip and particle, with the convention that the tip is grounded, and $2 \mathrm{e} / \mathrm{h} \approx 77 \mu \mathrm{S}$ is the conductance of a point contact [25]]. We use the zero temperature approximation and assume a constant density of states to find the simplified relation

$$
i(V, d)=\frac{2 e}{h} \int_{0}^{e V} e^{\frac{2 \sqrt{2}}{\hbar} \int_{0}^{d} \sqrt{m(U(x)-E} d x} d E
$$

We use a trapezoidal barrier with barrier height given by the work function of gold $\phi \approx 5 \mathrm{eV}$ and modified by a sinusoidal function which approximately accounts for image charging [26]]

$$
U(x)=e V\left(1-\frac{x}{d}\right)+\phi\left[\sin \left(\frac{\pi x}{d}\right)\right]^{\alpha}
$$

with the dimensionless parameter $\alpha=0.2$.

The current through the molecule $i_{\text {mol }}$ is given by the TRANSIESTA-C results as a function of the substrateparticle bias $V_{\text {mol }}=V_{\text {substrate }}-V_{\text {particle }}$ and the current through the air gap $i_{\text {gap }}$ by the WKB approximation as a function of the tip-particle bias $V_{\text {gap }}=V_{\text {particle }}-V_{\text {tip }}$ and the air gap $d$. In order to find the relation $i_{\text {tot }}\left(V_{\text {tot }}, d\right)$ for the currentvoltage characteristic of the entire system where $V_{\text {tot }}$ represents the bias between tip and substrate, we use $i_{\text {tot }}=i_{\text {mol }}=$ $i_{\text {gap }}=i$ and $V_{\text {mol }}+V_{\text {gap }}=V_{\text {tot }}$ for resistors in series. Rewriting with the current as independent variable, we have

$$
V_{\text {tot }}(i, d)=V_{\text {mol }}(i)+V_{\text {gap }}(i, d)
$$


which we again invert to find $i\left(V_{t o t}, d\right)$.

\section{Results and Discussion}

We find that the energetically most favorable geometry for the thiolates is with the sulfur atom between the fcc hollow and bridge sites (which we call the fcc/bridge site) at a height above the Au(111) slab of $2.1 \AA$ in the

GGA case and $2.0 \AA$ in the LDA case. The terminating carbon atom of DEB on the other hand sits $1.7 \AA$ above the $\mathrm{Au}(111)$ slab in the fcc hollow site [22]]. The choice of exchange correlation approximation has a significant effect on the interaction energies, but minimal effect on the equilibrium geometries. Current-voltage curves were found for all four molecules in this minimum energy geometry sandwiched between two gold electrodes by calculating the current at bias voltages between $-2.0 \mathrm{~V}$ and $2.0 \mathrm{~V}$ in steps of $0.1 \mathrm{~V}$, the results are given in Fig 3. The $0 \mathrm{~V}$ differential conductances for DTB, XYL, DEB and C12 are $40.8 \mu \mathrm{S}, 3.56 \mu \mathrm{S}, 10.9$ $\mu \mathrm{S}$ and $0.004 \mu \mathrm{S}$ respectively. For XYL, $i(V)$ curves were also calculated for the alternate adsorption site where the $S$ atom is between the ontop and hcp sites (denoted ontop/hcp in Fig 3.). The optimal height of the sulfur atom in this site was found as $2.4 \AA$ with SIESTA, calculations were performed at this optimal height as well as a height of $2.0 \AA$. The $0 \mathrm{~V}$ conductances in this case are $2.30 \mu \mathrm{S}$ and $1.58 \mu \mathrm{S}$ respectively. In addition, for XYL we test an alternate bonding geometry where the sulfur binds to the left electrode in the fcc/bridge site as above, but on the right electrode the S-H bond is not cleaved, geometry (b) in Fig 1. The sulphur-gold interaction in this case is much weaker and the optimal height of the sulfur is $2.4 \AA$. The corresponding $0 \mathrm{~V}$ conductance is $1.82 \mu \mathrm{S}$. These conductance values of XYL with different binding geometries are summarized in Table 1 together with the interaction energy of the molecule with the slab in each case.

Calculated currents for the geometry depicted in Fig 2, corresponding to an STS measurement of an electrode-molecule-nanoparticle-airgap, are shown in Fig 4. The current is plotted as a function of the air-gap for all three molecules at a total applied bias of $0.5 \mathrm{~V}$. For comparison, the result of a pure WKB calculation for an air-gap only with no molecule is also shown in Fig 4. 
Our current-voltage curve for DTB in the electrode-molecule-electrode configuration agrees well with that published by the developers of the code [8]]. The only notable difference is that the conductance in our calculation drops to half its $0 \mathrm{~V}$ value when the bias approaches $2.0 \mathrm{~V}$, rather than reach zero as in Ref. 8. This may be because of a different optimized geometry used.

In general the size of the current for the different molecules follows the interelectrode distances - a longer molecule results in a smaller current. It is interesting however that the current for DEB is about 5 times larger than for XYL, although the interelectrode distance for DEB is only slightly smaller. We propose that this is due to the unbroken conjugation arising from the alkynyl linkage to the gold surface. In thiolates, the S atom breaks this conjugation. We previously predicted that this molecule would form stable self-assembled monolayers on $\mathrm{Au}(111)$ [22] and it may thus be an interesting molecule to study in the context of single molecule conductivity.

Comparing the $G(0)$ values for XYL with different bonding geometries on the right electrode (see Table 1), shows that when the hydrogen atom is removed, the conductance follows the interaction strength, i.e. the geometries with higher interaction energies result in higher conductances. However, when the hydrogen atom is not removed, the conductance is high compared to the interaction energy. This could be because the change in stoichiometry leads to a different combination of orbitals being responsible for the conduction. Tao et al. [7]] measure $G(0)=0.046 \mu \mathrm{S}$, which is between one and two orders of magnitude smaller than our $a b$ initio results and the bonding geometries listed in Table 1 do not account for this. The introduction of a tunnel gap between a monolayer activated with gold nanoparticles and the electrode can drastically reduce the current as seen in Fig. 4. For each molecule we see initially a slow response as the gap is introduced where the resistance of the molecule dominates. However, as soon as the gap resistance dominates, the current decays exponentially with an increased gap distance $d$. This is especially apparent for the C12 molecule which is much longer and hence more resistive than the other molecules. Here the onset of exponential decay of current with gap distance only occurs at $d \sim 0.5 \mathrm{~nm}$. With this model, experimental and theoretical results can be reconciled for reasonably small gap distances, which are likely to be present in actual experiments. The shaded region in Fig. 4 indicates the range of experimental values found in the literature for DTB and XYL [4, 7, 27- 
29]]. The presence of such a gap is a realistic scenario in most experiments and this can obscure high conductances and interesting features in current-voltage characteristics of molecules. The results in Fig 4 also clearly show that for STS measurements to be able to distinguish between molecules as different as alkanethiols and alkynethiols the gap between tip and sample must be less than about $0.6 \mathrm{~nm}$. To distinguish between molecules with similar values of conductance, such as XYL and DTB, this gap must be less than about $0.2 \mathrm{~nm}$.

From the results in Fig 4 we can also gain some insight into the problem of measuring molecule heights in STM experiments. As analysed by Weiss et al. [30]] if an STM tip is scanned from a region of one type of molecule to another, or from a region of SAM to bare substrate the tip does not necessarily follow the true contours of the sample, but measures an apparent height. The reason for this is that the different regions will have different conductances and the tip will move to maintain constant current as well as follow the contours of the underlying surface.

We define $h_{S T M}$ as the height of the STM tip above the substrate, $h_{\text {mole }}$ as the thickness of the SAM, $d$ the gap distance between the STM tip and SAM and $\Delta h_{\text {STM }}, \Delta h_{m o l}$ and $\Delta d$ as the change in these quantities as the STM tip is moved at a fixed current from one type of molecule to another or to a clean surface. Then $\Delta h_{\text {STM }}$ $=\Delta h_{m o l}+\Delta d$. From Fig 4, if $d>0.6 \mathrm{~nm}$ then as the molecule moves from one molecule to another, for example from C12 to XYL, constant current can be maintained without altering $d$. In other words, $\Delta d=0$ and hence $\Delta h_{S T M} \approx \Delta h_{\text {mol }}$. In this regime the gap dominates the resistance and the difference in heights of the STM tip above the different molecules reflects the actual geometric height differences between the molecules. For tip-SAM distances less than $0.6 \mathrm{~nm}$ this is no longer the case and the apparent height deviates from the true height of the molecule. This is illustrated in Fig 5 for an STM tip scanning, at constant current, from a C12 molecule to a bare substrate. The apparent height measured by the STM minus the true height is plotted as a function of the set point current. For set point currents less than about $0.5 \mathrm{nA}$, which, from Fig 4, corresponds to a gap of $0.6 \mathrm{~nm}$ the apparent height is approximately equal to the true height. This is the regime described above. However, for currents greater than $0.5 \mathrm{nA}$, i.e. gaps $<0.6 \mathrm{~nm}$, the apparent height diverges rapidly from the true height. 
Fig 4 shows that if the tip is above a C12 molecule the tunneling current is approximately independent of the tip-molecule distance for distances less than about $0.6 \mathrm{~nm}$. In this regime if the tip now moves onto one of the other molecules studied here, the current can only be maintained by moving the tip to a gap of $0.51 \mathrm{~nm}$ above the molecule. This is inferred from Fig 4 by extrapolating the plateau region of C12 until it intersects the current-distance curves for the other molecules. If the tip started at a distance of $d_{C 12}$ above the C12 molecule then the change in tip height, or apparent height is $\Delta h_{S T M} \approx \Delta h_{m o l}+\left(0.51-d_{C 12}\right)$. In order to determine the true height of the molecule we would need to know the initial height of the tip above the C12 molecule.

\section{Conclusion}

We used the non-equilibrium Green's function method (NEGF) to calculate the $i(V)$ response of an electrodemolecule-electrode system for four different simple organic molecules. We found that using a non-optimal bonding geometry reduces the conductance considerably, but does not account for the orders of magnitude differences between theoretical and experimental conductances. As expected, the conductance is influenced by the length of the molecule, and falls very rapidly as the inter-electrode distance increases. Pi-conjugation within the molecule also appears to influence the tunneling current. Comparing two molecules with similar lengths, phenylene-dimethanethiol (XYL) and diethynylbenzene (DEB) the current differs by a factor of 5. We interpret this as due to the fact that in the former molecule $\pi$-conjugation is broken by the $\mathrm{S}$ atom whereas for DEB there is unbroken $\pi$-conjugation between the two electrodes giving a larger conductance. This result would suggest that DEB may be a worthwhile molecule for experimental molecular conductance studies.

We have also incorporated the effect of a tunnel gap in the configuration in order to simulate scanning probe measurements of molecular conductance. The configuration is now electrode-molecule-nanoparticlegap-probe tip. The tunnel gap was described using the Wentzel-Kramers-Brillioun (WKB) approximation and the remainder of the system using the NEGF formalism as above. In this case the calculated tunneling currents are in better agreement with available experimental results. This simple model also demonstrates that for gaps 
larger than about $0.6 \mathrm{~nm}$ scanning probe measurements are unable to distinguish between different molecules and will measure an identical $i(V)$ curve for any molecule.

\section{Acknowledgements}

This work was supported by the Australian Research Council and the University of Technology Sydney.

Computational resources were provided under the merit allocation schemes of the Australian Centre for Advanced Computing and Communications in New South Wales and the National Facility at the Australian Partnership for Advanced Computing.

\section{References}

[1] M. A. Reed, Proc. Ieee 87 (1999) 652.

[2] A. Nitzan and M. A. Ratner, Science 300 (2003) 1384.

[3] J. C. Love, L. A. Estroff, J. K. Kriebel, R. G. Nuzzo, and G. M. Whitesides, Chem. Rev. 105 (2005) 1103.

[4] M. A. Reed, C. Zhou, C. J. Muller, T. P. Burgin, and J. M. Tour, Science 278 (1997) 252.

[5] X. D. Cui, A. Primak, X. Zarate, J. Tomfohr, O. F. Sankey, A. L. Moore, T. A. Moore, D. Gust, G. Harris, and S. M. Lindsay, Science 294 (2001) 571.

[6] B. Q. Xu and N. J. J. Tao, Science 301 (2003) 1221.

[7] X. Y. Xiao, B. Q. Xu, and N. J. Tao, Nano Lett. 4 (2004) 267.

[8] K. Stokbro, J. Taylor, M. Brandbyge, J. L. Mozos, and P. Ordejon, Comp. Mater. Sci. 27 (2003) 151.

[9] H. Basch, R. Cohen, and M. A. Ratner, Nano Lett. 5 (2005) 1668.

[10] Y. B. Hu, Y. Zhu, H. J. Gao, and H. Guo, Phys. Rev. Lett. 95 (2005) 156803.

[11] G. C. Solomon, J. R. Reimers, and N. S. Hush, J. Chem. Phys. 122 (2005) 224502.

[12] K. H. Muller, Phys. Rev. B 73 (2006) 045403.

[13] G. C. Solomon, J. R. Reimers, and N. S. Hush, J. Chem. Phys. 121 (2004) 6615.

[14] C. Toher, A. Filippetti, S. Sanvito, and K. Burke, Phys. Rev. Lett. 95 (2005) 146402.

[15] P. Delaney and J. C. Greer, Phys. Rev. Lett. 93 (2004) 036805.

[16] N. Sai, M. Zwolak, G. Vignale, and M. Di Ventra, Phys. Rev. Lett. 94 (2005) 186810. 
[17] J. M. Soler, E. Artacho, J. D. Gale, A. Garcia, J. Junquera, P. Ordejon, and D. Sanchez-Portal, J. Phys.Condens. Matt. 14 (2002) 2745.

[18]P. Ordejon, E. Artacho, and J. M. Soler, Phys. Rev. B 53 (1996) 10441.

[19] N. Troullier and J. L. Martins, Phys. Rev. B 43 (1991) 1993.

[20] J. P. Perdew, K. Burke, and M. Ernzerhof, Phys. Rev. Lett. 77 (1996) 3865.

[21] C. Masens, M. J. Ford, and M. B. Cortie, Surf. Sci. 580 (2005) 19.

[22] M. J. Ford, R. C. Hoft, and A. McDonagh, Journal of Physical Chemistry B 109 (2005) 20387.

[23] M. Brandbyge, J. L. Mozos, P. Ordejon, J. Taylor, and K. Stokbro, Phys. Rev. B 65 (2002) 165401.

[24] J. P. Perdew and A. Zunger, Phys. Rev. B 23 (1981) 5048.

[25] S. Datta, Electronic transport in mesoscopic systems, Cambridge University Press, 1995.

[26] M. B. Cortie, M. H. Zareie, S. R. Ekanayake, and M. J. Ford, Ieee T. Nanotechnol. 4 (2005) 406.

[27] J. J. W. M. Rosink, M. A. Blauw, L. J. Geerligs, E. van der Drift, and S. Radelaar, Phys. Rev. B 62 (2000) 10459.

[28] M. Dorogi, J. Gomez, R. Osifchin, R. P. Andres, and R. Reifenberger, Phys. Rev. B 52 (1995) 9071.

[29] S. Datta, W. D. Tian, S. H. Hong, R. Reifenberger, J. I. Henderson, and C. P. Kubiak, Phys. Rev. Lett. 79 (1997) 2530.

[30] L. A. Bumm, J. J. Arnold, T. D. Dunbar, D. L. Allara, and P. S. Weiss, J. Phys. Chem. B 103 (1999) 8122. 


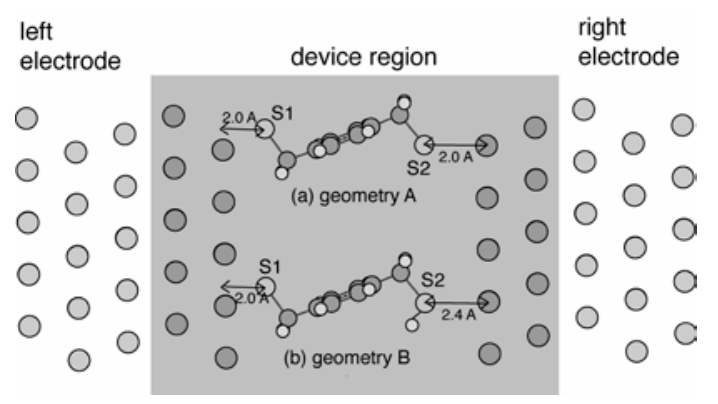

Figure 1: Computational setup used in TRANSIESTA-C calculations. XYL adsorbed between two Au(111) electrodes. On the left electrode the lowest energy geometry is used where the hydrogen atom is removed from the sulfur which binds to the gold surface at a height of $2.0 \AA$. On the right electrode we use (a) the same geometry and (b) the lowest energy geometry where the hydrogen is not removed from the sulfur. Here the sulfur binds at $2.4 \AA$ above the gold surface.

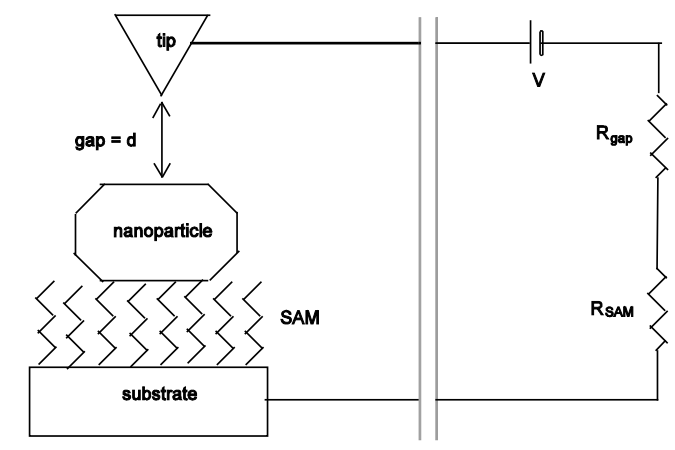

Figure 2: Schematic diagram of a scanning tunneling spectroscopy configuration. The resistance of the substrate-moleculenanoparticle part of the experiment is taken from the TRANSIESTA-C results for geometry A (see Fig. 1), and this is convoluted with a WKB approximation for tunneling through the gap between nanoparticle and tip to obtain the overall $i(V)$ response of the system.

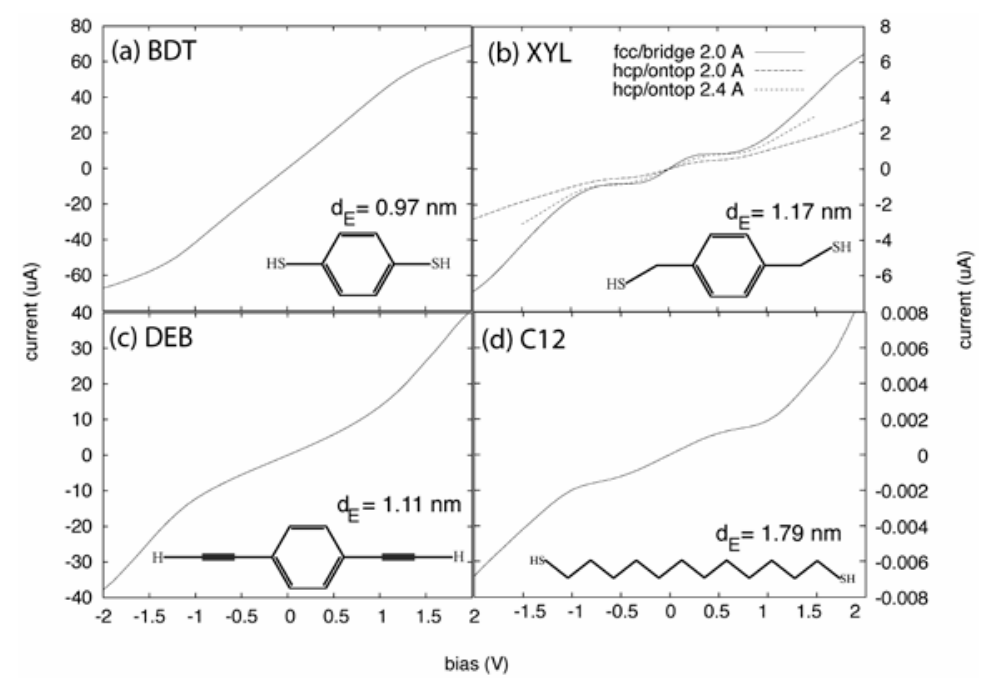


Figure 3: Current-voltage characteristics of the four molecules studied in the electrode-molecule-electrode configuration. The interelectrode distance is indicated for each molecule. For (b) XYL, three different bonding geometries are tested - the minimum energy geometry with the sulfur atom $2.0 \AA$ above the fcc/bridge site results in the largest current.

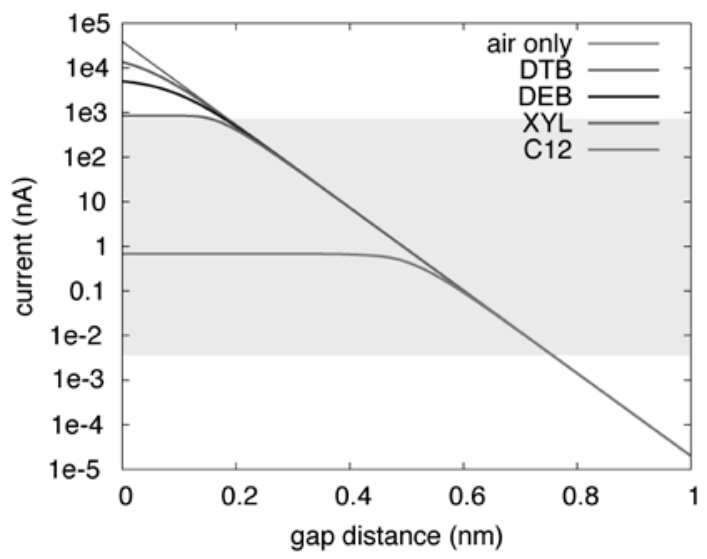

Figure 4: Current in the STS configuration as a function of tip-particle distance at an applied bias of $0.5 \mathrm{~V}$. The pure WKB tunneling current with no molecule present is also shown for comparison. The shaded region indicates the range of experimental $i(V=0.5)$ values for DTB and XYL found in the literature.

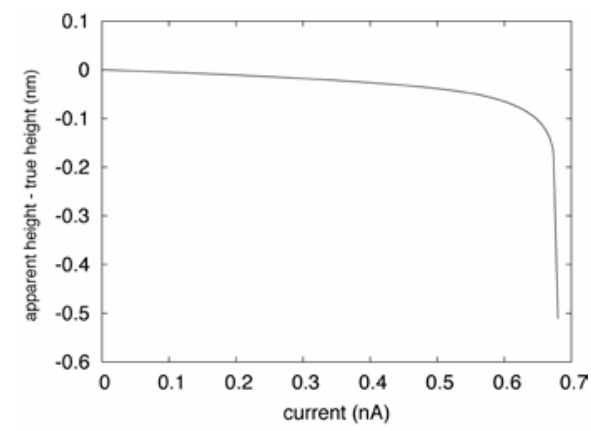

Figure 5: Offset of apparent height from the true height of a C12 molecule when an STM tip is moved from a self-assembled monolayer of C12 to a clean gold (111) slab.

\begin{tabular}{|c|l|l|l|}
\hline $\begin{array}{l}\text { binding site / } \\
\text { bond type }\end{array}$ & $\begin{array}{l}\text { S-Au(111) dist. } \\
\mathrm{d}(\AA)\end{array}$ & $\begin{array}{c}\text { interaction } \\
\text { energy }(\mathrm{eV})\end{array}$ & $\begin{array}{l}\mathrm{G}(0) \\
(\mu \mathrm{S})\end{array}$ \\
\hline fcc/bridge - S & 2.0 & -2.07 & 3.56 \\
\hline ontop/hcp - S & 2.0 & -1.26 & 1.58 \\
\hline ontop/hcp - S & 2.4 & -1.52 & 2.30 \\
\hline ontop/hcp - SH & 2.4 & -0.68 & 1.82 \\
\hline
\end{tabular}

Table 1. Zero-volt conductance and interaction energy for the interface geometries tested for XYL in the electrode-molecule-electrode configuration 